\title{
Le modèle didactique du genre à l'épreuve de la classe
}

Étude de la production écrite de trois élèves de $4 \mathrm{H}$ (CE1) en regard de leurs productions antérieures

The didactic model of "genre" according to the primary school classes. Analysis of three writing production of three pupils ( $4 \mathrm{H}$ level) compared to their previous ones

Véronique Bourhis, Danièle Frossard et Martine Panchout

\section{OpenEdition}

Journals

Édition électronique

URL : http://journals.openedition.org/pratiques/3374

DOI : $10.4000 /$ pratiques.3374

ISSN : 2425-2042

Éditeur

Centre de recherche sur les médiations (CREM)

Référence électronique

Véronique Bourhis, Danièle Frossard et Martine Panchout, « Le modèle didactique du genre à l'épreuve de la classe », Pratiques [En ligne], 173-174 | 2017, mis en ligne le 10 mars 2017, consulté le 14

novembre 2019. URL : http://journals.openedition.org/pratiques/3374 ; DOI : 10.4000/pratiques.3374

Ce document a été généré automatiquement le 14 novembre 2019.

(c) Tous droits réservés 


\section{Le modèle didactique du genre à l'épreuve de la classe}

Étude de la production écrite de trois élèves de $4 \mathrm{H}$ (CE1) en regard de leurs productions antérieures

The didactic model of "genre" according to the primary school classes. Analysis

of three writing production of three pupils ( $4 \mathrm{H}$ level) compared to their previous

ones

Véronique Bourhis, Danièle Frossard et Martine Panchout

1 Cet article se donne pour objectif de présenter quelques aspects de la production écrite d'élèves de l'école suisse romande en $4 \mathrm{H}$ ( $4^{\mathrm{e}}$ HarmoS, classe de fin du cycle 1 romand/ CE1, fin du cycle 2 en France). Ces productions résultent du travail conduit par une jeune enseignante qui suit sa classe depuis 3 ans $(2 \mathrm{H}=\mathrm{GS} ; 3 \mathrm{H}=\mathrm{CP}$ et $4 \mathrm{H}=\mathrm{CE} 1)$. Cette continuité nous permet d'observer longitudinalement la nature des matériaux dont le jeune scripteur fait usage : retrouve-t-on dans les productions des élèves les traces d'un « déjà là » dans leurs choix linguistiques, discursifs et stylistiques - et quelles sont ces traces ? Nous croisons nos regards, critiques et potentiellement contradictoires, sur ces productions écrites qui relèvent du genre « récit de vie ».

2 Nous posons d'abord les principes épistémologiques qui président à l'élaboration des curricula romands et sous-tendent les situations didactiques. En effet, la didactique du français à l'École primaire romande est construite en référence aux genres (Bakhtine, 1979 ; Dolz \& Schneuwly, 1998 ; Dolz, Noverraz \& Schneuwly, 2001), lesquels constituent l'arrière plan théorique du Plan d'Études Romand (PER, 2010). Selon cette posture épistémologique, le modèle didactique « désigne la description provisoire des principales caractéristiques d'un genre textuel en vue de son enseignement». Ainsi le modèle didactique du genre explicite «les savoirs de référence à mobiliser pour travailler les genres ; la description des différentes composantes textuelles spécifiques; les capacités langagières de l'élève qui les concernent » (Dolz \& Gagnon,2008). 
3 Nous contextualisons ensuite la production des textes dans ses aspects socioculturels et didactiques et présentons les productions écrites « libres» de trois élèves en fin de $4 \mathrm{H}$. Ces élèves ont été choisis en fonction du contraste de leur niveau initial lors des premières productions en $2 \mathrm{H}$ (MS).

4 À l'issue de cette entrée en matière nous interrogeons les productions en amont de ces mêmes élèves à l'oral et à l'écrit $(2 \mathrm{H} / \mathrm{GS}-3 \mathrm{H} / \mathrm{CP}-4 \mathrm{H} / \mathrm{CE} 1)$ et cherchons à identifier les traces des apprentissages effectués que l'on retrouve dans la dernière production.

Enfin, nous apprécions dans quelle mesure les élèves parviennent à se distancier ou non d'un schéma prototypique dont P. Bourdieu (1968), cité dans S. Juan (1999, p. 250) rappelle qu'il conduit forcément à une «simplification de la réalité réduite à des descripteurs ».

6 Nous terminons en montrant les forces et les limites de tels apprentissages, en portant une attention aux dimensions du genre, aux aspects graphiques et à l'engagement du sujet scripteur. Le PER prescrit de prendre en compte la « pensée créatrice ». En observet-on quelques traces?

7 Nous laissons volontairement de côté les questions cruciales de l'évaluation formative et des modalités de réécriture des élèves, ou encore de la formation initiale des enseignants à la didactique de la production d'écrit pour nous centrer sur une dynamique longitudinale.

\section{Fondements d'une didactique de l'écriture en Suisse romande: les genres}

\section{Les genres}

8 En didactique du français, la question des genres textuels est centrale. Elle ne fait cependant pas consensus : dans une étude diachronique, N. Denizot (2013) montre que les genres littéraires et textuels sont des constructions disciplinaires, qui servent différentes finalités selon les configurations scolaires : «ce sont à l'école des notions instables, dans leur définition, dans les corpus qui les actualisent ou dans les usages que l'on peut en faire ". Si les représentations hexagonales sont plutôt centrées sur la scolarisation des genres littéraires (Canvat, 1996) à travers lesquels différentes pratiques langagières sont susceptibles de s'incarner, ce sont les genres formels sociaux (Dolz \& Schneuwly, 1996) qui, scolarisés, constituent l'arrière plan théorique du PER 2010.

Cette dernière position prend appui sur la perspective socioculturelle qui souligne l'inscription de pratiques culturelles effectives dans une continuité (Vygotsky, 1985). Se référant à J.-P. Bronckart (1997), J. Dolz, et R. Gagnon (2008) soulignent que «tout membre d'une communauté est confronté à un univers de textes déjà là, univers organisé en genres empiriques et historiques, c'est à dire en formes d'organisation concrètes, susceptibles par ailleurs de se modifier avec le temps ». Les genres ont un ancrage social, une nature communicationnelle et répondent à des caractéristiques formelles, ce qui permet de distinguer des groupes de textes archétypiques liés par des régularités intertextuelles et répondant à des conditions de production spécifiques. 


\section{Les prescriptions institutionnelles}

10 grands types de conduites langagières : narrer, relater, argumenter, exposer et décrire des actions. Les textes relevant de la création poétique sont regroupés sur l'appellation «textes qui jouent avec la langue ». Chaque rubrique est intitulée Regroupement de genres, de telle sorte que l'élève puisse acquérir pendant sa scolarité des capacités langagières variées concernant différents genres relavant des différents regroupements.

En compréhension et production de textes, le PER préconise pour le regroupement de genres « le texte qui relate»:

- la carte postale, le récit de vie, le journal de bord pour le cycle 1 (1H-4H/MS-CE1);

- le récit de vie, le fait divers et l'esquisse biographique pour le cycle 2 (5H-8H/CE2-6 $\left.{ }^{\circ}\right)$.

En annexe 1, un tableau récapitulatif des instructions officielles du PER, concernant « la progression des apprentissages et des attentes fondamentales pour l'école primaire ». La classe de $6^{e}$ française correspond à la classe $8 \mathrm{H}$ romande, dernière classe de l'école primaire.

\section{Une conséquence : le modèle didactique du genre}

Faisant référence aux travaux de M. Bakhtine (1979), le genre est considéré comme un outil qui fonde les possibilités de toute communication, les «schèmes d'utilisation du genre ne référant pas seulement aux conditions habituelles dans lesquelles le genre fonctionne mais aussi aux conditions conçues et créées pour que le genre puisse fonctionner » (Schneuwly \& Dolz, 1997). description des principales caractéristiques d'un genre afin de pouvoir l'enseigner. Cette description se doit d'être évolutive, la modélisation «constitue une synthèse à visée pratique, destinée à orienter les interventions des enseignants " et "dégage les dimensions enseignables à partir desquelles diverses séquences didactiques peuvent être conçues » (Schneuwly et al., op. cit.). Elle s'effectue en référence aux finalités générales de l'école, au plan d'études dans tous les degrés du cursus scolaire.

Selon cette posture épistémologique, le modèle didactique du genre, appuyé sur une description des principales caractéristiques d'un genre donné, occupe « une place nodale dans le processus de transposition didactique » (De Pietro \& Schneuwly, 2003) et permet de structurer l'enseignement.

Ce modèle envisage la production d'écrit en termes d'opérations récursives dans ses aspects processuels, selon des étapes programmées qui supposent que le jeune scripteur mobilise un système qu'il s'est construit à partir de son expérience langagière singulière acquise au fil des apprentissages antérieurs.

dans le PER, il est recommandé d'aborder tous les regroupements de genres dès le premier cycle $(1 \mathrm{H}-4 \mathrm{H})$ tandis qu'au cycle 2 chacun d'eux fera l'objet d'un travail à deux reprises. Ceci signifie que chaque année il faut aborder au moins trois regroupements de genres pour se conformer à la conception spiralaire des apprentissages (Vygotsky, op. cit.) imposée par les programmes officiels. 


\section{Les dimensions du genre}

18 Pour chacun des regroupements de genres, le PER définit des objectifs se rapportant à différents niveaux fondamentaux d'opérations du fonctionnement langagier. Le genre étudié se caractérise par :

- la situation de communication: la représentation du contexte social (énonciateur destinataire, but, lieu social = domaine dans lequel s'inscrit le texte) ;

- le contenu : le sujet, les idées ;

- la planification : la structuration discursive du texte (mobilisation de la macrostructure du texte, du contenu sémantique global) ;

- la textualisation: le choix d'unités linguistiques (organisateurs verbaux et non verbaux: reprises pronominales et nominales, connecteurs, temps... titre, sous-titre, paragraphe, espace, illustration, marge...).

Deux autres dimensions enseignables et complémentaires aux précédentes sont la mise en page pour les productions écrites et les «moyens paralinguistiques» (prosodie, ton, pauses) pour les productions orales.

En situation de production, ces dimensions, issues du modèle d'analyse de textes proposée par J.-P.Bronckart (1997), sont critériées et permettent toute forme d'évaluation formative et/ou sommative.

On remarque des points de convergence entre le modèle de J. R. Hayes et L. Flower dont l'utilisation domine en France et le modèle d'analyse selon les 4 dimensions du genre utilisé en Suisse romande. Bien que J. R. Hayes et L. Flower placent la tâche de l'écriture dans son contexte psychologique, au carrefour entre la mémoire du rédacteur et l'environnement de la tâche à accomplir, et que celui des 4 dimensions du genre vise une analyse critériée qui spécifie des indicateurs, nous faisons un parallèle entre planification (Hayes \& Flower, 1980) et situation de communication, contenu et première ébauche de plan (modèle didactique du genre). Les opérations de mise en texte qui désignent chez J. R. Hayes \& L. Flower les activités liées à la production proprement dite correspondent à la planification et à la textualisation du modèle romand.

\section{La démarche didactique}

Elle suit une approche inductivo-déductive qui se rapproche du paradigme méthodologique de type explicatif - implicatif (Saada-Robert \& Leutenegger, 2002). Elle a été formalisée dans les Séquences didactiques: S'exprimer en français (Dolz, Noverraz \& Schneuwly, 2001), l'un des moyens d'enseignement romands. Cet outil, basé sur la modélisation didactique, est constitué de séquences. Chacune est consacrée à un genre. Elle débute par une production initiale, se termine par une production finale et est composée de modules que l'enseignant peut ajuster en fonction des besoins des élèves. Des critères et des outils d'évaluation liés au genre textuel étudié sont proposés. Selon ces perspectives et ces modalités didactiques, un "substrat» se constitue graduellement, et cette stratification de savoirs préside à toute nouvelle production d'écrit. Dans les faits, qu'en est-il ? 


\section{Conditions de production des textes : l'univers de l'écrit}

\section{Le récit de vie à l'école}

Selon Y. Reuter (2006, p. 220) qui cite M.-F. Bishop (2004), travailler à l'école les récits sollicitant le vécu présente des intérêts majeurs :

Ils appartiennent à une catégorie d'écrits inscrite dans la tradition scolaire [...]

même s'ils semblent plus lus qu'écrits dans les cadres pédagogiques classiques ;

- ils réclament des activités mentales au moins en partie différentes des écrits sollicitant l'imaginaire [...], et permettent une comparaison intéressante quant aux variations de la maîtrise narrative selon la relation au référent ;

- ils ont fait l'objet de multiples études (Bishop, 2004 ; Clanché, 1988 et 1992 ; Fayol, 1984 et 1985 ; Labov, 1978 ; Lahire, 1993) mettant au jour des problèmes tels que le rapport à la vérité, la sélection et la mémorisation des contenus, la mise en jeu de l'image de soi.

En suisse romande le récit de vie fait partie du regroupement de genres relevant des textes qui relatent (événement vécu) en opposition aux textes qui racontent (fiction).

\section{La classe : contexte socioculturel et didactique}

Nous avons suivi le travail d'une jeune enseignante nouvellement sortie de la Haute École Pédagogique (HEP) du canton de Vaud. Pour des raisons d'organisation interne de l'établissement scolaire auquel elle est rattachée, elle a suivi ses élèves durant trois ans. Cet établissement accueille une population importante issue de l'immigration.

Un rapport analysant le système de formation vaudois (Stocker, 2010, p.60-64) mentionne qu'en 1999, $35 \%$ des classes enfantines (MS et GS) et $27 \%$ des classes du primaire comptaient plus de $30 \%$ d'élèves allophones. Depuis lors, cette proportion ne cesse d'augmenter : en 2008-2009, elle était de $44 \%$ pour les classes enfantines et de $45 \%$ pour le primaire. Ces chiffres représentent une moyenne au niveau du canton mais dans l'établissement dans lequel travaille cette enseignante, la proportion d'élèves allophones atteint plus de $60 \%$.

Dans un tel contexte, le travail de l'oral constitue un axe d'intervention prioritaire (Verdelhan-Bourgade, 2007, p.54; Vigner, 2015, p.53), raison pour laquelle cette enseignante a mis en œuvre des dispositifs relevant de la didactique de l'oral en $2 \mathrm{H}$ (MS) et en $3 \mathrm{H}(\mathrm{CP})$. L'enseignante a travaillé sur le genre « récit de vie » en $2 \mathrm{H}$. Les séquences ont été filmées lors d'une recherche action de la HEP sur la mise en œuvre du dispositif « album écho " (Boisseau, 2005) à propos de goûters - les « récrés » en Suisse romande préparés à l'école.

En $3 \mathrm{H}(\mathrm{CP})$, l'enseignante a travaillé sur la capacité des élèves à reformuler une histoire entendue (réelle ou fictive). Le travail s'est focalisé sur les logiques temporelles et causales, avec usage des marqueurs « soudain » et « tout à coup ».

En 4H (CE1), elle s'est appuyée sur la démarche proposée par les séquences didactiques du manuel S'exprimer en français. La séquence concerne le récit de vie, dans sa modalité orale et s'adresse aux élèves du cycle 1 romand ( $\mathrm{cf}$. tableau ci-dessous). Ce moyen d'enseignement propose des activités et des séquences selon le modèle didactique du genre. L'enseignante a tiré parti d'une première visite à la ferme pour engager les élèves 
dans la production d'un récit oral et quelques mois plus tard, d'une seconde visite dans cette même ferme pour provoquer la rédaction d'un récit écrit.

De plus, en fin d'année scolaire $4 \mathrm{H}$ le «récit de vie " à l'oral a été l'un des objets de l'Épreuve cantonale de référence (ECR). Les compétences de compréhension d'un récit oral ont été validées par tous les élèves deux mois plus tard lors de l'ECR, ce qui mérite d'être souligné dans le contexte socioéconomique de cette classe.

Enfin, à la même période, un texte «libre " dont la consigne était de relater un souvenir partagé : la sortie au cinéma a été demandé aux élèves.

\section{Méthodologie}

Au fil des trois années $(2 \mathrm{H}$ à $4 \mathrm{H})$, toutes les productions d'élèves ont été recueillies: enregistrement des productions orales (fichiers audio), collecte des productions écrites. Nous avons sélectionné les productions de trois élèves dont le niveau initial était contrasté en début de scolarité $2 \mathrm{H}$ (MS), comme mentionné dans les propos introductifs.

Notre étude porte sur le corpus suivant : (annexe 2)

Tableau 1. Les productions des élèves

\begin{tabular}{|c|c|c|c|c|c|}
\hline \multicolumn{6}{|l|}{ Cycle 1} \\
\hline $2 \mathrm{H}(\mathrm{GS})$ & $3 \mathrm{H}(\mathrm{CP})$ & $\begin{array}{l}\text { 4H (CE1) } \\
\text { début d'année }\end{array}$ & $\begin{array}{l}\text { 4H (CE1) } \\
\text { début } \\
\text { d'année }\end{array}$ & $\begin{array}{l}4 \mathrm{H} \quad(\mathrm{CE} 1) \\
\text { milieu } \\
\text { d'année }\end{array}$ & $\begin{array}{l}\text { 4H (CE1) fin } \\
\text { d'année }\end{array}$ \\
\hline $\begin{array}{l}\text { Proto-récit de } \\
\text { vie oral }\end{array}$ & $\begin{array}{l}\text { Reformulation } \\
\text { d'un récit } \\
\text { entendu }\end{array}$ & Récit de vie oral & $\begin{array}{l}\text { Récit de vie } \\
\text { écrit }\end{array}$ & $\begin{array}{l}\text { Récit de } \\
\text { vie oral }\end{array}$ & $\begin{array}{l}\text { Récit de vie } \\
\text { écrit }\end{array}$ \\
\hline $\begin{array}{l}\text { L'album-écho: } \\
\text { «la semaine } \\
\text { des récrés » un } \\
\text { film } \\
\text { transcriptions }\end{array}$ & $\begin{array}{l}\text { «Les bêtises de } \\
\text { Chocolat Noir " } \\
\text { Entrainement à } \\
\text { l'usage de } \\
\text { « soudain " et } \\
\text { «tout à coup " } \\
\text { (pas de } \\
\text { production à } \\
\text { disposition) }\end{array}$ & $\begin{array}{l}\text { Le récit de vie } \\
\text { oral, séquence à } \\
\text { partir du manuel } \\
\text { Séquences } \\
\text { Didactiques, } 1 \text { ère } \\
\text { visite à la ferme : } \\
\text { enregistrements + } \\
\text { transcriptions }\end{array}$ & $\begin{array}{l}2^{\text {ème }} \text { visite à } \\
\text { la ferme } \\
\text { Productions } \\
\text { écrites }\end{array}$ & $\begin{array}{l}\text { évaluation } \\
\text { cantonale } \\
\text { (pas de } \\
\text { production } \\
\grave{a} \\
\text { disposition) }\end{array}$ & \begin{tabular}{|ll} 
production \\
spontanée \\
d'un récit de \\
vie écrit & (la \\
sortie & au \\
cinéma) &
\end{tabular} \\
\hline
\end{tabular}

\section{Présentation des productions libres manuscrites des trois élèves (4H, juin)}

Ci-dessous, les productions manuscrites du mois de juin en fin de cycle 1 (4H/CE1) et leur transcription littérale.

Une première lecture appelle probablement à chaud des commentaires que nous nous garderons de faire, réservant l'analyse dans notre $3^{\mathrm{e}}$ partie. 
Illustration 1. Ema : production libre manuscrite en fin de cycle
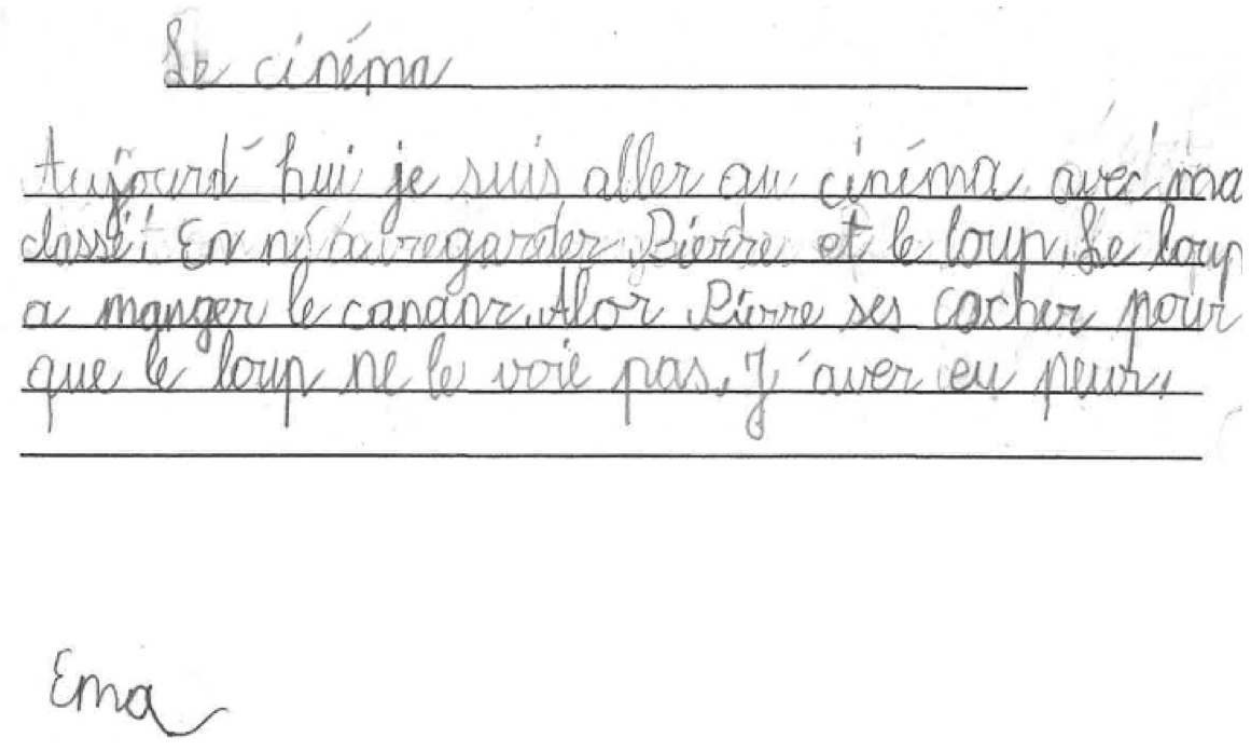

Le cinéma

Aujourd'hui je suis aller au cinéma avec ma classe ! On n'a regarder Pierre et le loup. Le loup a manger le canar. Alor Pierre ses cacher pour que le loup ne le voie pas. J'aver eu peur Ema

Illustration 2. Khawla : production libre manuscrite en fin de cycle

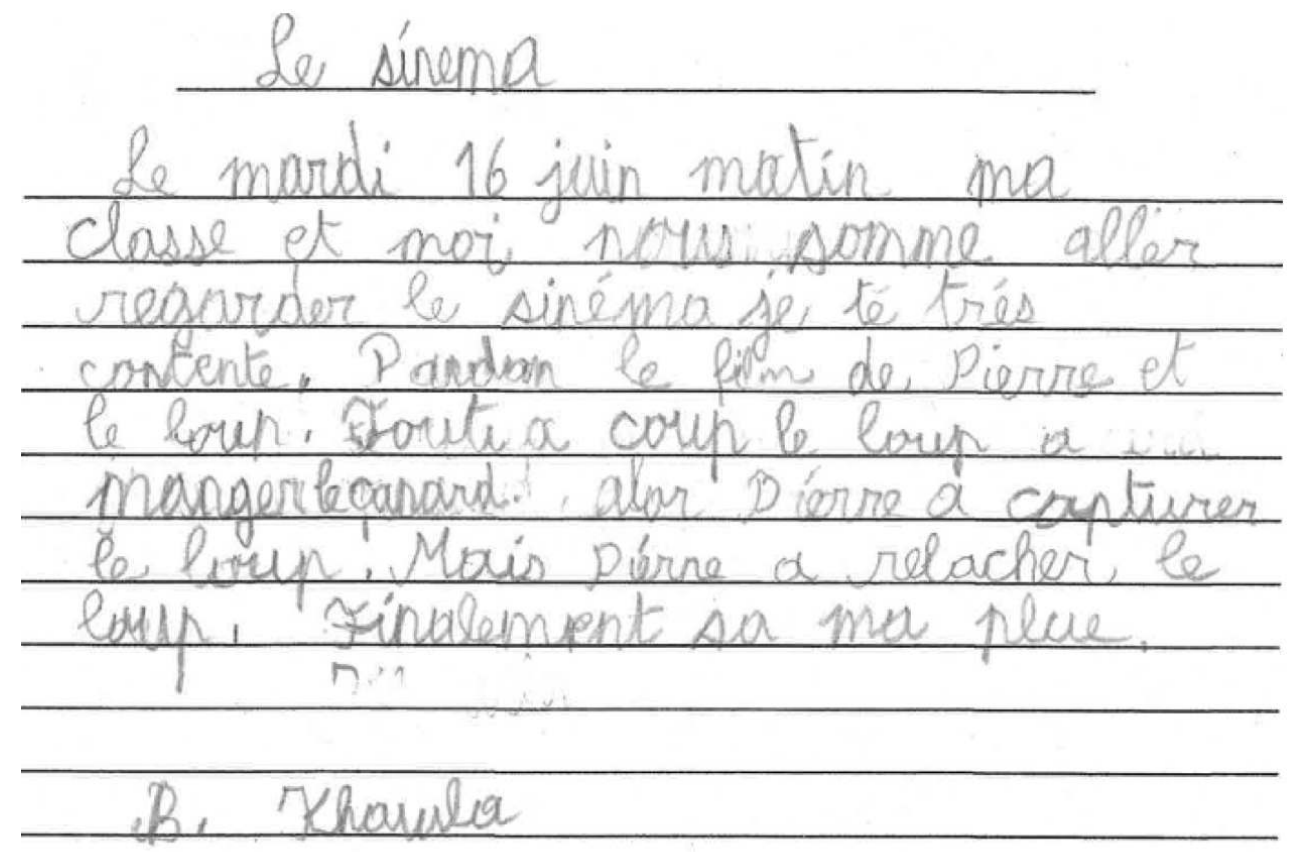

Le sinéma

Le mardi 16 juin matin, ma classe et moi nous somme aller regarder le sinéma je té très 
contente. Pandan le film de Pierre et

le loup. Toute a coup le loup a

manger le canard. alor Pierre a capturer

le loup. Mais Pierre a relacher le

loup. Finalement sa ma plue

B. Khawla

Illustration 3. Baptiste : production libre manuscrite en fin de cycle
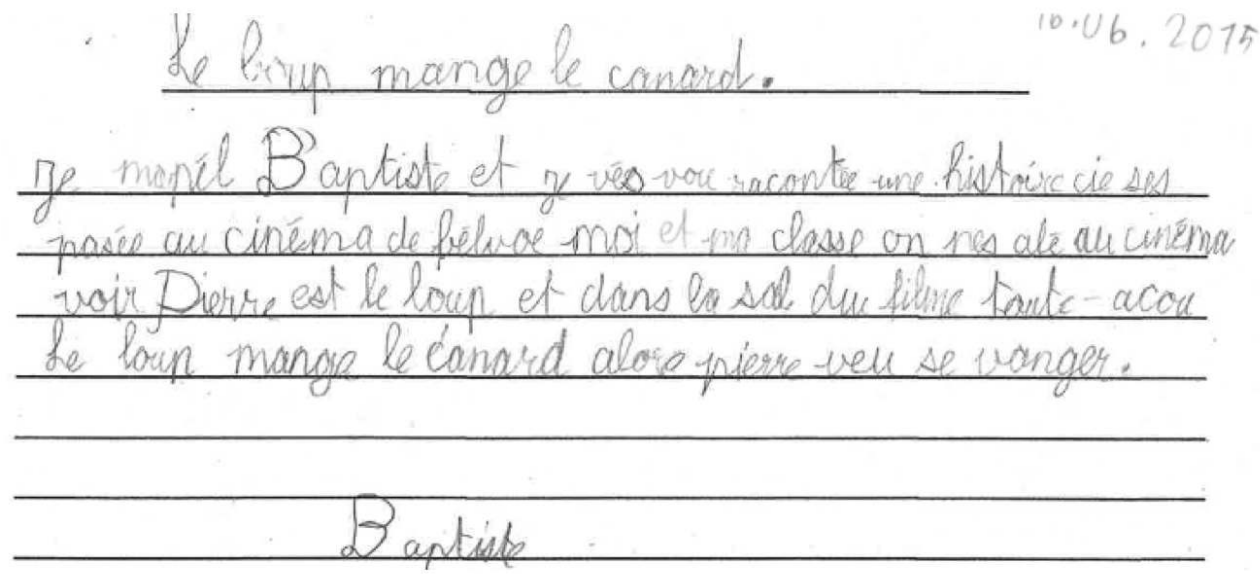

Le loup mange le canard

Je mapél Baptiste et je vés vou racontée une histoire cie ses

pasée au cinéma de bélvue moi et ma class on ne salé au cinéma

voir Pierre est le loup et dans la sal du filme toute acou

le loup mange le canard alors pierre veut se

venger.

Baptiste

Quelles traces d'un déjà-là dans les écrits spontanés répondant à la consigne « je relate la sortie au cinéma » de nos trois élèves de $4 \mathrm{H}$ (CE1) ? Quel parcours scriptural depuis la classe de $2 \mathrm{H}(\mathrm{GS})$ ? Telles sont les questions auxquelles nous allons tenter de répondre en examinant longitudinalement les productions orales et écrites des enfants.

\section{Le récit de vie au fil des classes et du temps : éléments d'analyse}

Notre analyse porte sur les dimensions enseignables telles que définies dans le modèle didactique du genre, formalisées par B. Schneuwly (1997), schématisées par J. Dolz et R. Gagnon (2008) et décrites précédemment.

\section{Les énoncés initiaux en $2 \mathrm{H}$}

Quelles sont les compétences initiales des enfants en $2 \mathrm{H}$ ?

Dans la séquence réalisée, l'activité langagière consiste en un proto-récit de vie sur support photos, avec un étayage langagier de la maitresse pour enrôler, maintenir, réduire les degrés de liberté (Bruner, 1998). Cette production verbale permet la réalisation d'albums échos (Boisseau, 2005) individuels (en je). Les paroles de l'enfant sont 
notées. Le projet est contextualisé, et la stratégie discursive circonscrite: les élèves "parlent» un récit de vie embryonnaire, premier pas vers la rencontre de régularités génériques et l'enseignant peut ainsi évaluer le rapport à la langue de chacun: dans le cadre de la semaine des "récrés", une infirmière est venue sensibiliser les élèves sur l'équilibre nutritionnel. Les albums échos relatent la décoration d'une boite pour mettre les goûters ainsi que le choix de différents produits adéquats du point de vue de la santé.

\section{Des récits référentiels}

Les trois productions présentent une unité de sens avec le genre, ce qui relève du contenu dans le modèle didactique. Les productions sont par ailleurs contraintes par les photos des albums-échos et l'étayage de l'enseignante.

On constate de grandes différences interindividuelles dans la production discursive. Alors qu'Ema commente avec beaucoup de difficultés, Khawla prend facilement la parole. Baptiste, quant à lui, a une relation élaborée à la langue et répond volontiers aux sollicitations.

Pour ce qui relève du lexique et de la syntaxe (textualisation), les difficultés d'Ema sont très importantes. Bien qu'elle soit en recherche lexicale (comment ça s'appelle?), le déficit de vocabulaire est marqué. Elle doit arrêter des choix qui sont problématiques :

Je sais pas qu'est ce que je fais (doublé d'une syntaxe inappropriée), j'sais pas comment ça s'appelle, je mets des mozzarella. Ses hésitations sont nombreuses.

Elle produit une construction paratactique et lie le personnage à l'action : $y^{\prime} a$ moi, je fais... Les premières formes syntaxiques complexes suivent rapidement sous la forme des relatives : y'a moi qui prends, y'a moi qui lève la main. En outre, les répétitions du présentatif (y'a moi, 7 occurrences) et de la mise en relief moi, je (3 occurrences) ont valeur de compensateur de cohésion et de marqueur de structure textuelle : y'a moi et Daniel, y'a encore le rhinocéros, y'a des pommes. Ces énoncés marquent une progression à thèmes éclatés.

47 Si Khawla emploie poire pour tomate (je sais pas comment il s'appelle), prononce la forme erronée infermier et n'a pas stabilisé l'utilisation des pronoms personnels : prenais gâteaux. prends pomme, elle utilise des phrases complexes (juxtaposition, coordination, connecteurs temporels).

48 Baptiste sait de quoi il parle et a les compétences lexico-syntaxiques pour le dire : infirmier - deviner - ingrédients - de la mozzarella.

\section{Énonciation, point de vue et modalisation (situation de communication - textualisation)}

49 Le discours est ancré dans la situation de communication. Tous les élèves utilisent la $1^{\text {re }}$ personne du singulier, la forme du pronom étant celle du personnel ou du pronom disjoint moi ou du clitique me. Ils utilisent également le nous.

Mais si les élèves marquent différemment leur implication au plan énonciatif, ils font cependant tous, à des degrés divers, la distinction entre discours et récit. Ema se centre essentiellement sur le commentaire des photos : «On fait des XX du travail sur le petit cahier »; «moi je vais prendre ça mais je sais pas comment ça s'appelle.» Deux occurrences marquent l'ici et maintenant : «moi, je me vois faire les petits points » et « mais je sais pas comment ça s'appelle ». Khawla s'implique ici et maintenant : « Pourquoi y'a 
pas nous? " « on voit Ethan, on voit... », tout comme Baptiste : «moi, je vois quelqu'un qui est moi », «Regarde » (il s'adresse à l'enseignante). Chaque enfant, hic et nunc, se pose donc en tant qu'énonciateur.

51 La valeur des temps verbaux est un bon indicateur des différences interindividuelles. Dans la textualisation des faits relatés, Ema choisit le présent : «j'choisis des autocollants », le présent progressif « on est en train de faire nos boites » et le futur périphrastique : «j'vais commencer ma boite, j'vais prendre ». Elle est dans l'action.

Khawla utilise d'abord le présent, mais lorsque l'enseignante lui demande : "qu'est-ce que t'étais en train de faire? » elle répond en utilisant également l'imparfait. Khawla est capable de marquer la décentration qu'implique le passé.

Baptiste emploie le présent d'énonciation: "je me vois ", "c'est moi qui est en train de dessiner ». Les interjections Oh la la! Mama mia, la la la la la la, les onomatopées pffffff et les rires qui ponctuent son discours confère à celui-ci une coloration affective, modalités d'énoncé qui traduisent l'engagement de l'enfant et l'expression de la subjectivité énonciative. Par la suite, lors d'une relance de l'enseignante il utilise pertinemment l'imparfait, l'imparfait progressif, "Françoise était en train de nous montrer les ingrédients », le plus que parfait à valeur d'antériorité « je les avais mis dans ma boite». On observe même une glose métadiscursive au conditionnel « Comment je pourrais dire ça?»

\section{Des productions initiales contrastées}

Si l'on se place du point de vue de la cohérence, du choix des mots, on remarque que les moyens linguistiques des élèves sont contrastés.

L'enseignante attend un proto récit de vie avec un déroulement chronologique. Or les élèves décrivent strictement le référent. Toutefois, ils utilisent déjà tous quelques (rares) connecteurs temporels : «On fait du travail sur le petit cahier. Et puis, là, on est en train de faire nos boites. " (Ema). "Je prends ma boite et puis je suis debout." «J'étais debout pour prendre ma boite et pour faire une photo. » (Khawla) « et aussi, j'vois Andrei » «J'étais en train de couper les fruits et je les avais mis dans ma... comment ça s'appelle déjà ? Dans ma boite. Ensuite je te regardais faire ça. » (Baptiste).

La construction de la cohérence est difficile d'autant que peu de phénomènes locaux marquent la cohésion. L'isotopie sémantique n'est pas facile à saisir pour un observateur extérieur qui n'a pas les photos sous les yeux : Ema a des difficultés à construire des liens entre son expérience et les savoirs langagiers que l'enseignante essaie de lui faire utiliser. Les difficultés de Khawla sont plutôt d'ordre syntaxico-lexical. Les marques de cohésion interphrastique sont assurées et une première syntaxe du révolu acquise. Quant à Baptiste, il relate la semaine des récrés sans réelles difficultés langagières, même si des formes erronées subsistent. Il manipule plusieurs formes temporelles dont il maîtrise les valeurs aspectuelles.

Dans ces productions orales basées sur l'album écho, aucune contrainte textuelle n'est imposée. Les dimensions du genre telles que définies dans le modèle didactique sont enseignées implicitement : situation de communication, valeur des temps, déroulement chronologique. 


\section{Le récit de vie à l'oral}

\section{L'enseignement du récit oral en $3 \mathrm{H}$ et début de $4 \mathrm{H}$}

Pour rappel, en 3H, les préparations de l'enseignante indiquent qu'elle a travaillé sur la capacité des élèves à reformuler un récit entendu (Les bêtises de Chocolat Noir). Le travail s'est centré sur une restitution chronologique et logique des faits avec usage des marqueurs « soudain » et « tout à coup ».

En $4 \mathrm{H}$, elle s'est appuyée sur la séquence didactique du manuel S'exprimer en français: le récit de vie à l'oral. Chacune de ces séquences est organisée selon le schéma ci-dessous :

Illustration 4. Organisation de la séquence didactique

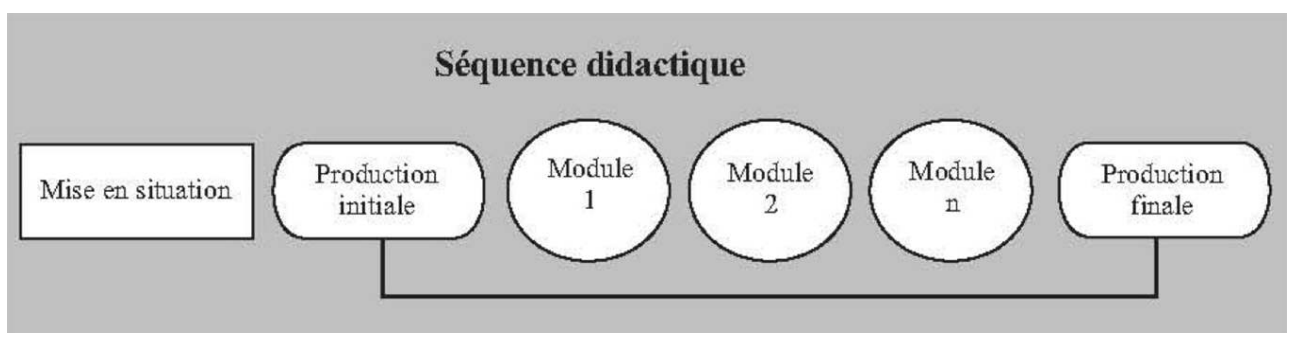

Dans la séquence consacrée au récit de vie, il est attendu des élèves qu'ils mettent en œuvre des «opérations de cadrage » qui assurent la prise de contact avec l'auditoire : présentation de soi, salutations et explicitation de l'objet du discours.

Après une production initiale orale permettant une évaluation diagnostique, des modules sont proposés.

En fonction des besoins des élèves, les notions suivantes ont été spécifiquement enseignées :

- Situation de communication : texte en je / présence du « toi » ou « vous ».

- Planification : présence de trois parties.

- Contenu: présence d'une formule introductive; "narration» (relater) d'un événement particulier.

- Textualisation : présence de reprises; respect du temps des verbes ; présence de discours indirect ; mise en voix : intonation variée, débit adéquat, volume adapté.

La production finale est enregistrée.

\section{Les élèves s'appuient-ils sur les apprentissages antérieurs ?}

4 Dans le cadre de la production d'un récit de vie à l'oral (4H/CE1), les élèves ont eu pour consigne de relater un "évènement inattendu » survenu lors d'une visite scolaire à la ferme.

Pour alléger la charge cognitive des élèves, l'enseignante a proposé pour la production orale une grille de soutien à la parole, adaptée au texte à produire ainsi qu'une feuille organisée en trois espaces sur lesquels dessiner trois moments du récit (annexes 3 et 4). Ces outils permettent à l'élève de centrer son discours sur des particularités génériques concernant différents niveaux d'organisation.

- Le respect des contraintes issues de la prescription : de nouveaux acquis scolaires 
On note d'abord le respect des contraintes matérielles : le récit de vie à l'oral est enregistré. Sa production en est facilitée par le recours aux guides de production (planification et demande d'effectuation de trois dessins) - annexes 3 et 4 . Ces supports permettent aux élèves de parler leurs récits de manière fluente, de les organiser à la « lecture des dessins ».

Intervient ensuite le respect des contraintes textuelles.

Les trois productions orales se caractérisent par une homologie macrostructurelle. Au niveau de la situation de communication, elle est marquée par les formules de cadrage du discours bonjour ; je m'appelle; au revoir et par l'interpellation du destinataire en début et fin de récit:je vais vous raconter, j'espère que mon histoire vous a plu. Au plan de la planification et de la textualisation, chaque paragraphe est marqué par des pauses et englobe une signification - unique pour un ensemble d'éléments rendue compréhensible par les tout à coup et alors.

69 Le modèle didactique engendre la production d'une forme fixe stéréotypée, liée à l'appartenance générique.

- Un modèle au service du déjà là

Pour autant, il favorise l'expansion des acquis déjà là par récursivité. Il est l'objet d'une double sémiotisation : c'est d'abord un objet d'étude que les élèves doivent s'approprier mais en même temps il crée de nouvelles significations que les élèves élaborent à partir du déjà là initial, notamment dans la construction de la cohérence.

71 Si la posture énonciative en «je » et la présence des deux énonciations discours-récit apparait déjà en $2 \mathrm{H}$, la visée spécifique du modèle induit la production d'un certain nombre de caractères qui relèvent de la cohésion référentielle, de la cohésion verbale, de l'articulation de la progression thématique, de la présence des connecteurs. Chaque anecdote relatée est présentée de manière intelligible et constitue un authentique récit (planification et textualisation). Le parcours interprétatif de l'écouteur peut se construire dans une dynamique basée sur la reconstitution de l'anecdote relatée :

Ema: Franco raconte une histoire / Ema voit une araignée / Elle détourne la tête et écoute l'histoire ;

Khawla : Les enfants se promènent dans un champ de maïs / ils se perdent / l'animateur soulève un camarade de classe / ils rentrent à la ferme ;

Baptiste: Les enfants vont voir des lapins / celui que porte Baptiste s'échappe / il le rattrape et le met dans sa cage.

Mais le lecteur doit parfois activer des hypothèses dans un double mouvement anticipation-rétroaction de lecture. Rien ne précise le lien entre l'effet suscité par la vue de l'araignée et l'action qui suit: j'ai retourné la tête (Ema), ou encore entre le fait que l'animateur soulève un élève (pour voir dans quelle direction se trouve la ferme ?) et que tous puissent rentrer.

La cohérence textuelle se construit pas à pas.

Le manuel S'exprimer en français. Séquences didactiques pour l'oral et pour l'écrit indique que le genre étudié à l'oral consiste en la production d'« un événement inattendu venant rompre une tension initiale ; Il s'agit d'un récit de vie contenant une tension narrative. » (Préambule p. 44, séquence 2 récit de vie, cycle 1). Une forme de tension narrative, est présente dans les trois productions : l'araignée fait très très peur, les enfants se sont perdus 
, un lapin s'est échappé. L'expression de la subjectivité est cependant peu présente : seule Khawla utilise la relative : une histoire que j'ai vécue à la ferme, qui marque une implication plus personnelle que l'expression une histoire qui s'est passée à la ferme.

Ainsi, en début de $4 \mathrm{H}$, les productions des élèves à l'oral reflètent un déjà-là consécutif à la sédimentation des apprentissages précédents, réactivé par la confrontation au modèle dont l'enseignement constitue un levier pour la production.

\section{Le passage à l'écrit}

\section{Les productions écrites en $4 \mathrm{H}$}

- Une linéarisation difficile

Confrontés à la page, les élèves ont à gérer des opérations procédurales très complexes : concevoir l'écrit dans sa globalité (situation de communication, contenu, organisation des propos, mise en mots).

Les productions montrent que la mise en mots, transformation des « résultats émanant de l'élaboration conceptuelle du message » en une "forme linguistique » (Garcia-Debanc \& Fayol, 2002, p. 41), ne va pas de soi alors même que les compétences de textualisation sont présentes à l'oral (cf productions précédentes). Rappelons que les compétences de compréhension d'un récit oral ont été validées par tous les élèves deux mois plus tard lors de l'ECR. C'est donc que la contrainte imposée par la consigne ainsi que le schéma prototypique du genre déjà travaillé depuis deux mois ont constitué pour les élèves des ressources. Qu'implique le passage à l'écrit?

81 Pour écrire leur texte, les élèves bénéficient d'une page comportant trois espaces numérotés, dans lesquels dessiner les étapes de l'anecdote choisie, suivis de trois blocs de lignes coïncidant avec les paragraphes attendus (annexe 4). Les illustrations recèlent des détails qui enrichissent le récit et partagent l'instance narrative entre texte et dessins, ce qui témoigne d'une créativité des élèves dans la gestion de la consigne et est susceptible de renforcer l'investissement subjectif des auteurs.

Un premier constat s'impose: les productions sont nettement moins denses que les productions orales, ce qui s'explique compte tenu du phénomène de linéarisation qu'implique le traitement écrit.

83 Toutefois, si l'on laisse de côté le phénomène de surface que constitue l'orthographe, que nous livrent ces premiers écrits?

- De la planification globale à la planification locale

$84 \mathrm{Du}$ point de vue de la situation de communication, les trois producteurs de textes adoptent la posture énonciative attendue : je, nous, on à valeur de nous. Il s'agit d'un récit de vie écrit, donc aucun d'eux n'explicite formellement qu'il s'agit de relater un évènement vécu lors de cette seconde visite à la ferme - ce qui serait attendu lors d'un récit oral. Pourtant deux d'entre eux, mentionnent pertinemment le contexte de cette visite.

Le déjà là macrostructurel (contenu, planification) rythme la production : une anecdote réelle est racontée. Comme pour la production orale précédente, la macrostructure des trois textes est identique. Et les productions respectent les métarègles de cohérence textuelle: les organisateurs textuels et connecteurs créent la relation de congruence entre les éléments (tout à coup est généralisé, alors est présent pour deux élèves), les 
répétitions sont assurées par des pronoms anaphoriques sujets ou COD (elle, on, l'...); l'information est reprise de façon logique et se renouvelle de façon continue. Enfin il n'y a pas de contradiction entre l'univers que le texte propose et les choix énonciatifs, temporels et logiques des élèves.

Mais alors que précédemment ( $4 \mathrm{H}$ oral) le modèle didactique créait de nouvelles significations dans la construction de la cohérence, il génère ici localement trois nouveaux types de significations qui lui sont liées.

- La ponctuation : elle n'est pas toujours utilisée de manière phrastique et son usage n'est pas normatif, notamment dans la production d'Ema. Pour autant, est-elle aléatoire ? V. Paolacci et M. Favart (2010) cite C. Fabre (1989) et M. Fayol (1989) : les signes sont produits dès les débuts de l'écrit, au CE1 $(4 \mathrm{H})$. Le point est le signe majoritairement utilisé. Selon l'auteur, «ce type de planification [...] consiste, pour le rédacteur débutant, à s'adapter au coût cognitif de la rédaction en appliquant à l'écrit le système de l'oral. Il opère alors par cycles de traitement, chaque cycle étant constitué de la récupération d'un contenu en mémoire suivie de sa production. On note alors une juxtaposition de propositions séparées éventuellement par des points. " Ema opère par scripts, entendus comme une suite de propositions, chacune décrivant un événement situé à l'intérieur d'une séquence chronologique sans que les faits entretiennent forcément de relation de succession. Elle surponctue, ce qui permet de marquer en surface des regroupements et des séparations de blocs d'événement ou d'état. Maris noël. Une fermier qui socuper de nou. Elle à sorti la pizza. Du four. Les propositions adjacentes peuvent présenter un degré de liaison plus ou moins élevé selon qu'elles relèvent ou non du même épisode. M.-J. Béguelin (2000) identifie un phénomène de sous-ponctuation dans les écrits d'enfants que l'on trouve également dans la production d'Ema, les deux phénomènes semblant ne pas s'exclure mutuellement : et mari noël a dit « ou la la » j'avai eu peur. C'est la seule qui marque un discours direct et utilise des guillemets.

Khawla maitrise mieux les signes ponctuants. Ses phrases commencent par une majuscule et se terminent par un point, elle utilise la virgule à bon escient : tout à cous on à glisé de la montagne de niee, on à u tré peur. Elle sous-ponctue aussi : .... on a gimpé on haut de la montagne de nieg on été tré conten.

Baptiste n'a pour sa part oublié qu'une majuscule : le car de la ferme est venu nou chercher.

- Les effets stylistiques : les élèves essaient tous de produire une certaine tension narrative, déjà là depuis le récit oral. Outre les aspects lexicaux, ce sont les phénomènes de cohésion verbale qui la suscitent : Ema relate les faits au passé composé. Le seul plus que parfait : javai eu peur constitue une glose à valeur métadiscursive, que renforcent le lexique : alarme, peur, la présence d'un discours direct «Hou, là, là !» ainsi que l’onomatopée «Bip Bip » dans le deuxième dessin.

Dans le récit au passé composé de Khawla, l'usage des imparfaits à valeur durative pour exprimer un ressenti on été conten est tout à fait judicieux par opposition au passé composé on à u tré peur qui traduit l'expression immédiate d'un plaisir-crainte délicieux lié à une prise de risque... modérée.

Pour Baptiste, l'incident du car qui transporte les élèves et glisse un peu sur la neige est perçu comme drôle et est présenté sur un mode humoristique, avec la présence d'un discours direct dans l'illustration: Allez, allez! Alors que le premier paragraphe alterne passé composé et imparfait (on et tré conten est à lire phonétiquement : était), l'insertion du présent constitue une rupture qui renforce le suspense et qui crée un effet de 
proximité avec le lecteur : les actions sont présentes dans la mémoire du narrateur et paraissent plus vivement ressenties.

- La clôture du texte : Les trois textes sont clos. La clôture de Ema est prévisible car isotope avec l'événement relaté : alors marie-noël a attinte (éteint) l'alarme. Celle de Khawla est appréciative et conclusive : Et on été tré conten. Quant à Baptiste, son texte se termine par le mot Fin, ce qui clôt définitivement le procès. Bien que non adaptée aux dimensions du genre, cette transgression est intéressante du point de vue de la posture métadiscursive du scripteur.

\section{Les productions « libres»}

91 Que se passe-t-il quand les élèves produisent librement un récit de vie? La seule consigne donnée est : « je relate la sortie au cinéma ». Pour cette production l'enseignante n'a explicité aucune contrainte ni rappelé aucun critère relevant des quatre dimensions du genre à produire.

Quelles formes scripturales sont stabilisées et relèvent de la restitution des savoirs langagiers des élèves? L'écrit demeure-t-il dans un format et une énonciation conforme au modèle didactique ? L'élève s'éloigne-t-il de la reproduction des stéréotypes langagiers et à quel niveau de la production : sémantique, textuel, discursif ?

- La consigne

Alors que le terme « récit de vie » n'est pas employé, la formulation de la consigne n'est pas neutre: «je relate la sortie au cinéma». Le verbe, généralement inusité dans ce contexte, a manifestement été choisi par l'enseignante pour que les élèves produisent ce genre étudié maintenant depuis trois ans. Le « je » impose une énonciation particulière.

On peut s'interroger sur le fait que dans les programmes helvètes le récit de vie est présenté comme faisant partie d'un autre regroupement que celui des textes qui racontent. Dans le préambule de S'exprimer en français. Séquences didactiques pour l'oral et pour l'écrit, on lit (p. 44): «en ce qui concerne le contenu des récits, la priorité a été donnée à la vraisemblance plutôt qu'à la vérité. En effet, on peut concevoir que tous les élèves n'aient pas vécu des évènements dignes d'être relatés. Ce qu'ils auront à raconter, ce seront alors des histoires susceptibles de leur être arrivées. On n'exclut pas dans certains cas que l'élève se projette dans l'imaginaire, pour autant qu'il présente son aventure du point de vue de celui qui vit les évènements (utilisation du JE) ».

Si le récit de vie est une narration en « je » en quoi diffère-t-il des autres récits narratifs ? Cette distinction entre monde réel et monde fictionnel est pour le moins ambiguë.

- Organisation spatiale et mise en page Tous les textes ont un titre, le cinéma, celui de Baptiste s'écartant délibérément de la consigne : le loup mange le canard.

L'écriture en tant que geste graphique est réalisée au crayon à papier et sans interligne Seyes, ce qui est tout à fait inhabituel en France pour des CE1 mais normal en Suisse où l'on attend que l'écriture cursive normée soit apprise jusqu'en $4 \mathrm{H}$. La ligne unique est fréquente à partir de la $5 \mathrm{H}$.

98 Les productions sont assez courtes, si l'on excepte le titre et la signature : 4 lignes pour celles d'Ema et de Baptiste, 8 lignes pour Khawla qui, du reste, écrit plus gros. 

manifestent.
Les productions, visibles en manuscrit au point 2.4 - ne respectent pas une mise en paragraphes liée à la présence des connecteurs, comme la superstructure du modèle l'impose : les élèves ont écrit leur production sous une forme compacte.

Bien que les élèves aient plusieurs fois rencontré la superstructure conventionnelle et qu'ils s'y soient conformés, elle n'est pas complètement stabilisée. Ils ne la projettent pas sur les macrostructures sémantiques.

- Les quatre dimensions du genre

En surface, les critères correspondant aux 4 dimensions du genre sont respectés. Comme dans les productions précédentes, les énoncés sont en "je » et les élèves relatent un événement vécu (situation de communication). Le "contenu » est circonscrit par la consigne : la sortie au cinéma. Au plan de l'organisation du texte (planification), toutes les productions sont balisées par des connecteurs, notamment tout à coup et alors. Les textes d'Ema et de Khawla comportent une phrase introductive. Au niveau de la textualisation (mise en mots), toutes les deux produisent des phrases syntaxiquement complètes et bien ponctuées. Paradoxalement Baptiste sous-ponctue son texte - il se clôt seulement d'un point final, alors que sa production précédente était normée.

Il faut également noter que, contrairement à la France, l'attention des enfants n'est pas attirée sur l'importance de la norme orthographique au motif que l'élan créatif risquerait d'être entravé par une vigilance orthographique excessive dont l'élève sait déjà qu'il ne saurait la respecter. Ce qui est susceptible de causer une hypovigilance que les textes

Les productions écrites sont donc relativement homogènes quant à leur contenu et présentent toutes une forme de stéréotypie: nature du message, longueur, type de discours, temps verbaux, thème..., ce qui signifie que le modèle didactique permet aux élèves les moins performants de mettre en mots, à l'instar des autres élèves, sans rester bloqués sur un des aspects de la production d'écrit.

- Les initiatives du sujet scripteur

Les trois productions reflètent bien la difficulté du genre. En effet, les élèves ont intégré que le récit de vie doit "accrocher» le lecteur et proposer une certaine tension narrative : le texte doit avoir une visée perlocutoire. Que relatent-ils ? Comme le note M.F. Chanfrault-Duchet (1983), le je-sujet d'un récit de vie ne peut être un je-énonciateur, puisque le temps verbal utilisé est le passé composé. Dans leurs productions, les trois élèves tiennent plus compte du destinataire que de l'aspect formel du fait relaté puisqu'ils ciblent ce qui les a intéressés et ce qui est susceptible d'intéresser le lecteur. Ce n'est pas tant le rapport qu'ils entretiennent avec leur vie passée qui leur importe que la relation qu'ils instaurent avec le lecteur à l'occasion de leur discours sur le passé. Ainsi chacun raconte le récit de fiction « Pierre et le loup » et particulièrement le moment crucial où le loup mange le canard. Le fait relaté devient le récit cadre dans lequel s'enchâsse le récit du film, la narration est mise en abîme dans le « fait relaté ».

Au plan de la cohérence, on repère aisément les principales isotopies des productions de Khawla et d'Ema : la sortie scolaire ; l'histoire de Pierre et le loup ; l'expression d'un point de vue modal. L'entrelacs donne une certaine densité au texte.

La production de Baptiste est plus singulière : il s'écarte du modèle initial par le titre qu'il donne à sa production : le loup mange le canard. Mais il y revient dès la $1^{\text {re }}$ ligne après s'être présenté : je m'appelle Baptiste et je vais vous raconter une histoire qui s'est passée au 
cinéma de Bellevue. Moi et ma classe on est allé au cinéma voir Pierre et le loup et dans la salle du film tout à coup le loup mange le canard alors Pierre veut se venger. Le texte de Baptiste est un récit de vie modélisé jusqu'au moment où un brouillage des topics produit une allotopie qui rompt la cohérence du texte : et dans la salle du film tout à coup le loup mange le canard alors Pierre veut se venger. Cependant si l'on considère à la suite de F. Rastier (1985) que "ce n'est pas la récurrence et la combinatoire des sèmes déjà donnés qui constitue l'isotopie mais au contraire la présomption d'isotopie qui permet d'actualiser les sèmes ", alors le principe de cohérence est bien respecté (ce qui se passe hors de la salle de cinéma / ce qui se passe dans la salle: le film). Baptiste cherche manifestement à produire une tension narrative qu'il ne trouve pas dans la sortie elle-même !

La « pensée créatrice » est prescrite par le PER selon trois descripteurs : le développement de la pensée divergente, la reconnaissance de sa part sensible, la concrétisation de l'inventivité.

Ema et Khawla expriment leurs sentiments : j'avais eu peur, j'étais très contente, finalement ça m'a plu. Pour autant, cette expression est encore très limitée : les élèves s'en tiennent aux procédés déjà utilisés. Baptiste ose la pensée divergente : il s'affranchit du stéréotype, sait varier sa source d'inspiration et exprime ses idées sous de nouvelles formes.

\section{Conclusion}

109 Le genre est un outil sémiotique de l'activité langagière. L'analyse des productions à partir d'une pratique d'enseignement effective montre que le modèle didactique, dont la finalité se veut heuristique, comporte une dimension prescriptive prégnante tant dans les gestes professionnels de l'enseignante que dans les processus scripturaux des élèves - les deux étant par ailleurs liés.

110 Cependant, si l'on tient compte du niveau langagier initial très contrasté des élèves (proto-récit $2 \mathrm{H}$ ), l'analyse longitudinale des productions écrites montre également que le modèle didactique réactualise le déjà là dans la mémoire de travail et qu'il favorise indubitablement la production écrite des élèves les moins performants.

111 Enfin, s'il n'autorise que peu l'émergence d'une pensée créatrice qui engage le sujet scripteur et s'il ne peut s'affranchir de la stéréotypie - le risque étant que les élèves fassent une homologie entre le produit et les processus de production, le modèle didactique du genre permet néanmoins d'envisager la production comme un acte de composition récursive, autant latérale que linéaire.

\section{BIBLIOGRAPHIE}

BAKHTINE, M. (1979). Esthétique de la création verbale. Trad. du russe par Alfreda Aucouturier. Paris : Gallimard.

BÉGUELIN, M.-J. (2000). De la phrase aux énoncés. Grammaire scolaire et descriptions linguistique. Bruxelles : De Bœck Duculot. 
BISHOP, M.-F. (2004). Les écritures de soi à l'école primaire de 1850 à 2004. Place, fonctions et enjeux. Thèse de doctorat : Université Charles-de-Gaulle-Lille 3. BOISSEAU, P. (2005). Enseigner la langue orale en maternelle. Paris : Retz. Bourdieu, P., CHABOREDON, J.-C. \& PASSERON J.-C. (1968). Le métier de sociologue. Paris : Mouton/ Bordas.

BRONCKART, J.-P. (1997). Activité langagière, textes et discours. Lausanne : Delachaux et Niestlé.

BRUNER, J. (1998). Le développement de l'enfant : savoir faire, savoir dire. Paris : Presses universitaires de France.

CANVAT, K. (1996). « Types de textes et genres textuels. Problématique et enjeux ». Enjeux 37/38, p. 5-30.

CHANFRAULT-DUCHET, M.-F. (1983). « L'énonciation et les ruses du sujet ». Revue des sciences humaines 192, p. 99-107.

DE PIETRO, J.-F. \& SCHNEUWLY, B. (2003). « Le modèle didactique du genre : un concept de l'ingénierie didactique ». Recherches en didactique. Les Cahiers Théodile 3, p. 27-52.

DENIZOT, N. (2013). La scolarisation des genres littéraires (1802-2010). Bruxelles : P. Lang, 2013.

DOLZ, J. \& SCHNEUWLY, B. (1996). « Genres et progression en expression orale et écrite. Éléments de réflexions à propos d'une expérience romande ». Enjeux 37/38, p. 49-75.

- (1998). Pour un enseignement de l'oral : initiation aux genres formels de l'oral. Paris : ESF éditeur.

DOLZ, J, NOVERRAZ, M. \& SCHNeUWly, B. (2001). S'exprimer en français. Séquences didactiques pour l'oral et pour l'écrit. Bruxelles : De Boeck.

DOLZ, J. \& GAGNON, R. (2008), « Le genre du texte, un outil didactique pour développer le langage oral et écrit ». Pratiques 137-138, p. 179-198.

DOLZ, J. \& DUFAYS, J. L. (2012). « Les didactiques des disciplines à l'heure du curriculum ». In : Carlier, G., De Kessel, M., Dufays, J.-L., Wiame, B. (dirs), Progressions et transversalité. Comment (mieux) articuler les apprentissages dans les disciplines scolaires? Louvain : Presses universitaires de Louvain, p. 78-92.

FABRE, C. (1989). « Les débuts de la ponctuation au CP ». Études de linguistique appliquées 73, p. 59-70.

FAYOL, M. (1989). « Une approche psycholinguistique de la ponctuation. Étude en production et en compréhension ». Langue Française 81(1), p. 21-39.

GARCIA-DEBANC, C. \& FAYOL, M. (2002). « Apports et limites des modèles du processus rédactionnel pour la didactique de la production écrite. Dialogue entre psycholinguistes et didacticiens ». Pratiques 115-116, p. 37-50.

HAYES, J. R. \& FLOWER, L. (1980). « Identifying the organization of writing processes ». In : Gregg, L. W. \& Steinberg, E. R. (éds), Cognitive Processes in Writing. Hillsdale, NJ: L. Erlbaum, p. 3-30.

JUAN, S. (1999) Méthodes de recherche en sciences sociohumaines. Paris : Presses universitaires de France.

LEUTENEGGER, F., \& SAADA-ROBERT, M. (éds) (2002). Expliquer et comprendre en sciences de l'éducation. Bruxelles : De Boeck supérieur. 
PAOLACCI, V. \& FAVART, M. (2010). « Traitement des marques de cohésion par les jeunes scripteurs : l'utilisation de la ponctuation et des connecteurs à l'entrée en sixième. Approche linguistique, cognitive et didactique ». Langages 177(1), p. 113-128.

RASTIER, F. (1985). «L'isotopie sémantique, du mot au texte ». L'Information Grammaticale 27(1), p. 33-36.

REUTER, Y., (2006). « Les récits sollicitant le vécu au CM2. Éléments d'analyse et de comparaison ». Repères 34, p. 111-139.

SCHNEUWLY, B. \& DOLZ, J. (1997). « Les genres scolaires. Des pratiques langagières aux objets d'enseignement ». Repères 15, p. 27-40.

STOCKER, E. (2010). Analyse du système de formation vaudois à l'aide d'indicateurs. Lausanne : URSP.

VERDELHAN-BOURGADE, M. (2007). Le français langue seconde. Un concept et des pratiques en évolution. Bruxelles : De Boeck.

VIGNER, G. (2015). Le français langue seconde. Comment apprendre le français aux élèves nouvellement arrivés. Paris : Hachette Éducation.

VyGotsky, L. (1985) [1934]. Pensée et langage. Trad. de Françoise Sève. Paris : La Dispute, Paris.

\section{ANNEXES}

\section{Annexe 1. Récit de vie et PER « Le texte qui relate »}

\begin{tabular}{|c|c|c|c|}
\hline CYCLE 1 & $1 \mathrm{H}-2 \mathrm{H}$ & $3 \mathrm{H}-4 \mathrm{H}$ & Attentes fondamentales \\
\hline $\begin{array}{l}\text { Compréhension } \\
\text { de textes }\end{array}$ & $\begin{array}{l}\text { Compréhension d'un } \\
\text { récit lu par l'adulte } \\
\text { (carte postale, récit de } \\
\text { vie, journal de bord...) }\end{array}$ & $\begin{array}{l}\text { Compréhension individuelle } \\
\text { d'un récit (carte postale, récit } \\
\text { de vie, journal de bord...) : } \\
\text { repérage de la suite des } \\
\text { événements du récit; } \\
\text { perception des sensations et } \\
\text { des sentiments des } \\
\text { personnages }\end{array}$ & \multirow[b]{2}{*}{$\begin{array}{l}\text { l'élève comprend le sens } \\
\text { global d'un texte } \\
\text { narratif et situe les } \\
\text { événements }\end{array}$} \\
\hline $\begin{array}{l}\text { Production } \\
\text { de textes }\end{array}$ & $\begin{array}{l}\text { Création en groupe } \\
\text { ou individuellement, } \\
\text { avec l'aide de } \\
\text { l'adulte, d'un récit } \\
\text { (récit de vie, carte } \\
\text { postale...) }\end{array}$ & $\begin{array}{l}\text { Rédaction en groupe ou } \\
\text { individuellement d'un texte } \\
\text { qui relate des événements } \\
\text { réels (récit de vie, carte } \\
\text { postale...) : distinction des } \\
\text { différentes parties d'un texte } \\
\text { (formule de politesse, date, titre, } \\
\text { signature...) ; utilisation de } \\
\text { quelques organisateurs } \\
\text { temporels (un jour, une nuit; } \\
\text { ensuite, après, alors; tout à } \\
\text { coup, soudain...) }\end{array}$ & \\
\hline
\end{tabular}




\begin{tabular}{|c|c|c|c|}
\hline \multicolumn{2}{|c|}{$\begin{array}{l}\text { MER (moyen } \\
\text { d'enseignement }= \\
\text { manuel) }\end{array}$} & $\begin{array}{l}\text { S'exprimer en français : le récit } \\
\text { de vie }\end{array}$ & \\
\hline CYCLE 2 & $5 \mathrm{H}-6 \mathrm{H}$ & $7 \mathrm{H}-8 \mathrm{H}$ & Attentes fondamentales \\
\hline $\begin{array}{l}\text { Genres } \\
\text { conseillés }\end{array}$ & le récit de vie & $\begin{array}{l}\text { le fait divers et l'esquisse } \\
\text { biographique }\end{array}$ & \multirow{8}{*}{$\begin{array}{l}\text { l'élève écrit un texte } \\
\text { respectant le genre } \\
\text { travaillé (situation, lieu, } \\
\text { personnages, } \\
\text { chronologie des } \\
\text { événements) et } \\
\text { intégrant des passages } \\
\text { dialogués (paroles } \\
\text { rapportées directement } \\
\text { ou indirectement) }\end{array}$} \\
\hline \multicolumn{3}{|c|}{$\begin{array}{l}\text { Création d'un univers de réalité en utilisant ses connaissances } \\
\text { scolaires et extrascolaires } \\
\text { Choix d'un titre }\end{array}$} & \\
\hline & & $\begin{array}{l}\text { Écriture du chapeau d'un fait } \\
\text { divers }\end{array}$ & \\
\hline \multicolumn{3}{|c|}{$\begin{array}{l}\text { Distinction entre parties qui relatent et parties dialoguées } \\
\text { Insertion de paroles rapportées directement ou indirectement pour le } \\
\text { fait divers }\end{array}$} & \\
\hline \multicolumn{3}{|c|}{$\begin{array}{l}\text { Utilisation d'organisateurs temporels (un jour, hier, le lendemain...), } \\
\text { d'accélérateurs de rythme (soudain, tout à coup...), de la ponctuation } \\
\text { relative au dialogue et de verbes de parole }\end{array}$} & \\
\hline & & $\begin{array}{l}\text { Utilisation d'organisateurs } \\
\text { logiques (et alors, si bien que...) }\end{array}$ & \\
\hline \multicolumn{3}{|c|}{$\begin{array}{l}\text { Utilisation adéquate des oppositions (imparfait/passé composé, } \\
\text { présent/passé composé) }\end{array}$} & \\
\hline MER & & $\begin{array}{l}\text { S'exprimer en français : le fait } \\
\text { divers }\end{array}$ & \\
\hline
\end{tabular}

\section{Annexe 2. Transcriptions}

\begin{tabular}{|l|l}
\hline Album écho $(2 \mathrm{H})$ oral & Récit de vie oral (début de $4 \mathrm{H})$
\end{tabular}


E: Qu'est-ce que tu vois Ema?

Ethan, Sarah avec le doudou... et Daniel et moi.

E : Qu'est-ce que vous êtes en train de faire?

On fait des..X du travail sur le p'tit cahier

Et pis là, on est en train de faire nos boites

Y a moi. Euh... euh...j'vais... j'vais commencer ma boite.

Y a moi, j'choisis des autocollants pour mettre sur ma boite. $14: 18$

Y a moi et Daniel... moi je choisis des autocollants...

Moi, je me vois faire les petits points. $\mathrm{Y}$ a encore le rhinocéros

J'mets des légumes dans ma boite. $Y$ a des pommes... qui sont... pommes séchées. Et des... et des biscuits. Y a moi qui qui prends des biscuits

Moi j'vais prendre ça, mais j'sais pas comment ça s'appelle... (Ethan souffle : des abricots secs) des abricots secs.

J'sais pas qu'est-ce que je fais... Je mets des mozzarella

Y a moi qui lève la main et Thomas et Daniel

Bonjour Je m'appelle Ema et je vais vous raconter une histoire qui s'est passée à la ferme. (7s) Ma classe et moi nous sommes parties à la ferme. C'était deux jours et une nuit (13s).

Franco, un fermier qui nous faisait à manger, nous avait nous avait raconté une histoire le soir avant de dormir. Tout à coup, j'ai retournée la tête et j'ai vu une araignée qui dansait et une araignée qui me faisait très très peur. Alors, j'ai retourné la tête sans penser aux araignées et j'ai écouté l'histoire de Franco ; au revoir ! J'espère que mon histoire vous a plu! 
Pourquoi y a pas nous? Comme moi j'fais des cœurs

On voit Ethan, on voit Sarah, on voit Daniel, on voit moi. (gestes) On est en train de colorier. C'est moi

Qu'est-ce que tu es en train de faire? (photo où elle montre sa boite, de face) En train de regarder ma boite. J'ai perdu les lettres, je prends ma boite et puis je suis debout. J'ai en train de faire ma boite. Je fais des cœurs.

Je fais une photo.

C'est moi qui t'ai pris en photo mais qu'est-ce qu't'étais en train de faire?

J'étais debout pour prendre ma boite et pour faire une photo.

Qu'est-ce que tu nous montrais? Je montrais ma boite

(Photo où elle choisit les biscuits) ça, c'est moi.

Et qu'est-ce que tu faisais Rhawla? prenais gateaux.

Qu'est-ce que tu fais là R. ? Prends... prends pomme chessée (séchées)

Tu me regardes (en parlant à J.)

Et là ? Je coupe la pomme

Alors ici? Qu'est-ce que tu es en train de faire? Je en train de... je prends une poire tu prends une poire? je ne sais pas comment il s'appelle...

L'ens demande aux autres élèves et J. propose " tomate».

Je mets la mozzarella. C'est comme du fromage

Et ici qu'est-ce qu'on voit ? l'infermière
Bonjour, je m'appelle Khawla et je vais vous raconter une histoire que j'ai vécue à la ferme. Un lundi après-midi, mon /mes camarades de classe et moi, nous sommes allés se promener dans les champs de maïs. Tout à coup, nous nous sommes perdus. Alors, Bruno, celui qui s'occupait de nous, a soulevé un des élèves de classe qui s'appelle Haron. Enfin, nous avons pu entrer à la ferme. J'espère que mon histoire vous a plu

BAPTISTE 
Qu'est-ce que tu es en train de faire? Je fais une photo de ma boite et ch'suis tout rouge.

Oh là, là ! Ma ma mia ! C'est moi qui est en train de dessiner avec XX (du ro ?) ma boite.

moi j'vois qqch qui est moi (sur photo de groupe)

Baptiste, qu'est-ce que tu vois? $\mathrm{j}$ ' vois moi XXXX j'me vois moi en train de faire des dessins, la la la, la-la la puis j'vois Joana en train de faire sa boite... Regarde : comme ça, et aussi j'vois Andrei qui regarde sa boite pour dessiner? j'vois Yelena aussi qui cherche des crayons, un crayon qu'que part.

(autre photo) Rires C'est moi en infirmier... Qu'est-ce que t'es en train de faire? en train d'choisir mes trucs de récré infirmier pour la récré infirmier.

Pourquoi t'étais en infirmier?_. Pffff ! parce que je suis un infirmier... d'ailleurs j'jouais avec Andrei

Photo suivante... Qu'est-ce que t'es en train de faire? J'ai en train d'te regarder... J'ai en train de... j'avais choisi c'que j'voulais... quatre biscuits...

(Autre photo) Qu'est-ce que tu étais en train de faire? j'étais en train de couper les fruits, et j'les'avais mis dans ma... comment ça s'appelle déjà dans ma boite, merci Yelena (qui lui avait soufflé), ensuite je te regardais en train de faire comme ça...

Photo suivante J'ai en train de mettre des tomates dans la boite de ma maman,

photo suivante moi en train de mettre des tomates... et de la mozzarella

En parlant de la photo avec l'infirmière: Françoise était en train de nous montrer les ingrédients, les... comment j'pourrais dire ça ? que on pourrait manger à la récré...

Récit de vie écrit, début de $4 \mathrm{H}$
Bonjour, je m'appelle Baptiste, je vais vous raconter quelque chose qui s'est passé à la ferme.

Mardi, avec l'école, nous sommes allés dormir une nuit à la ferme. Avec Sissi, la maman d'Aurélie, notre maitresse, nous sommes allés voir les lapins. Et, j'en ai porté un. Quand TOUT A COUP, le laPIN a ssauté. Alors je l'ai rattrapé et tout de suite mis dans sa cage.

Au revoir ! Merci d'avoir écouté mon histoire! 


\begin{tabular}{|c|c|}
\hline $\begin{array}{l}\text { Mari noël. Une fermier qui } \\
\text { socuper de nou . Elle à sorti } \\
\text { la pizza. Du four. } \\
\text { tout à cou l'alarme à soner. } \\
\text { Et mari noël a dit « ou la la « } \\
\text { javai eu peur. } \\
\text { Alors mari noël à aitinte l'alarme. }\end{array}$ & $\begin{array}{l}\text { Le cinéma } \\
\text { Aujourd'hui je suis aller au cinéma avec ma } \\
\text { classe! On n'a regarder Pierre et le loup. Le loup } \\
\text { a manger le canar. Alor Pierre ses cacher pour } \\
\text { que le loup ne le voie pas. J'aver eu peur } \\
\text { Ema }\end{array}$ \\
\hline \multicolumn{2}{|l|}{ KHAWLA } \\
\hline $\begin{array}{l}\text { Moi et mes camar de classe nous som } \\
\text { alle à la ferme } 2 \text { jour et une nuit. } \\
\text { Un lundi apré-midi moi et mes } \\
\text { camarad de class, on à gimpé on haut } \\
\text { de la montagne de nieg on été tré conten. } \\
\text { Tout à cous on à glisé de la montagne de } \\
\text { niege, on à u tré peur. } \\
\text { On est arivé on bat de la mentag de nieg. } \\
\text { Et on été tré conten. }\end{array}$ & $\begin{array}{l}\text { Le sinéma } \\
\text { mardi } 16 \text { juin matin, ma } \\
\text { classe et moi nous somme aller } \\
\text { regarder le sinéma je té très } \\
\text { contente. Pandan le film de Pierre et } \\
\text { le loup. Toute a coup le loup a } \\
\text { manger le canard. alor Pierre a capturer } \\
\text { le loup. Mais Pierre a relacher le } \\
\text { loup. Finalement sa ma plue } \\
\text { B. Khawla }\end{array}$ \\
\hline \multicolumn{2}{|l|}{ BAPTISTE } \\
\hline $\begin{array}{l}\text { Je suis allé à la ferme. le car de la ferme est } \\
\text { venu nou chercher. Après on nét prèsc arié } \\
\text { avec ma classe. } \\
\text { Tou-acou le car de la ferme glic. Et set drole. } \\
\text { Alor on la encourager et on né ariv à la } \\
\text { ferme. } \\
\text { fin }\end{array}$ & $\begin{array}{l}\text { Le loup mange le canard } \\
\text { Je mapél Baptiste et je vés vou racontée une } \\
\text { histoire cie ses } \\
\text { pasée au cinéma de bélvue moi et ma class on ne } \\
\text { salé au cinéma } \\
\text { voir Pierre est le loup et dans la sal du filme } \\
\text { toute acou } \\
\text { le loup mange le canard alors pierre veut se } \\
\text { venger } \\
\text { Baptiste }\end{array}$ \\
\hline
\end{tabular}




\section{Annexe 3. Guide de production du récit de vie à l'oral} $(4 \mathrm{H})$

Le récit de vie

Je dis bonjour

- J'explique de quoi je vais parler

Début de mon récit :

Je donne assez d'informations : - - Q - Quand

Où

- Quoi
- Avec qui

Comment

Milieu et fin de mon récit :

Quelque chose arrive, j'utilise tout à coup

J'utilise Alors pour expliquer comment j'ai réagi.

- Petite phrase pour finir ma présentation

- Je dis au revoir 
Annexe 4.1. Productions écrites de Baptiste et d'Ema en appui sur le guide d'écriture (1)

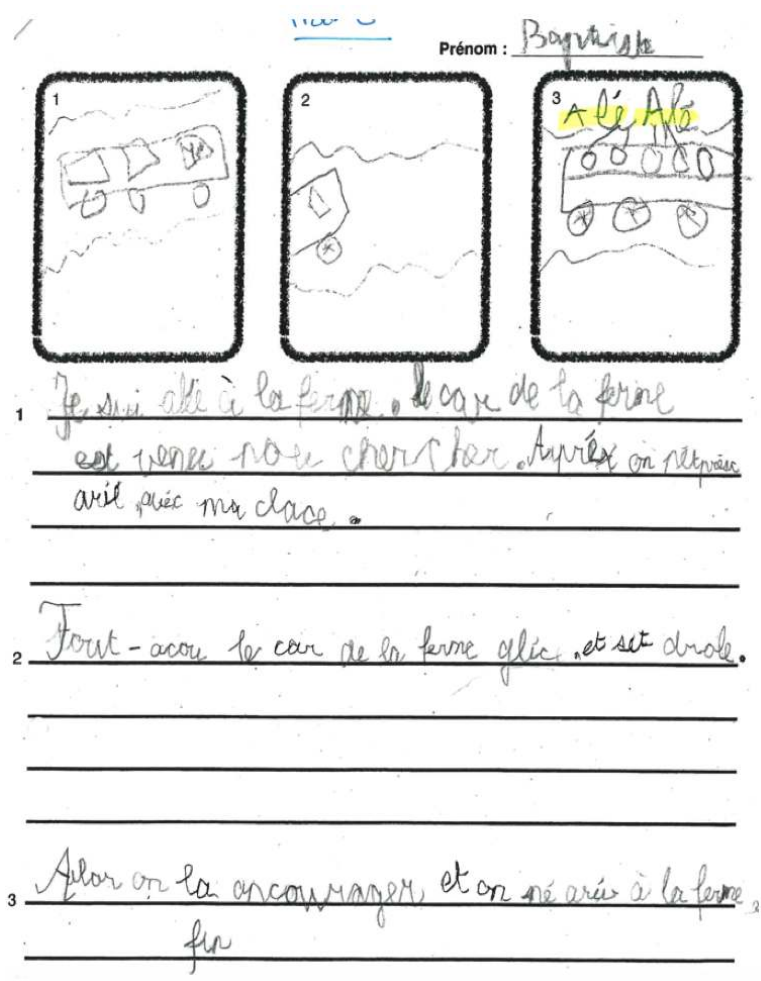




\section{Annexe 4.2. Productions écrites de Baptiste et d'Ema en appui sur le guide d'écriture (2)}

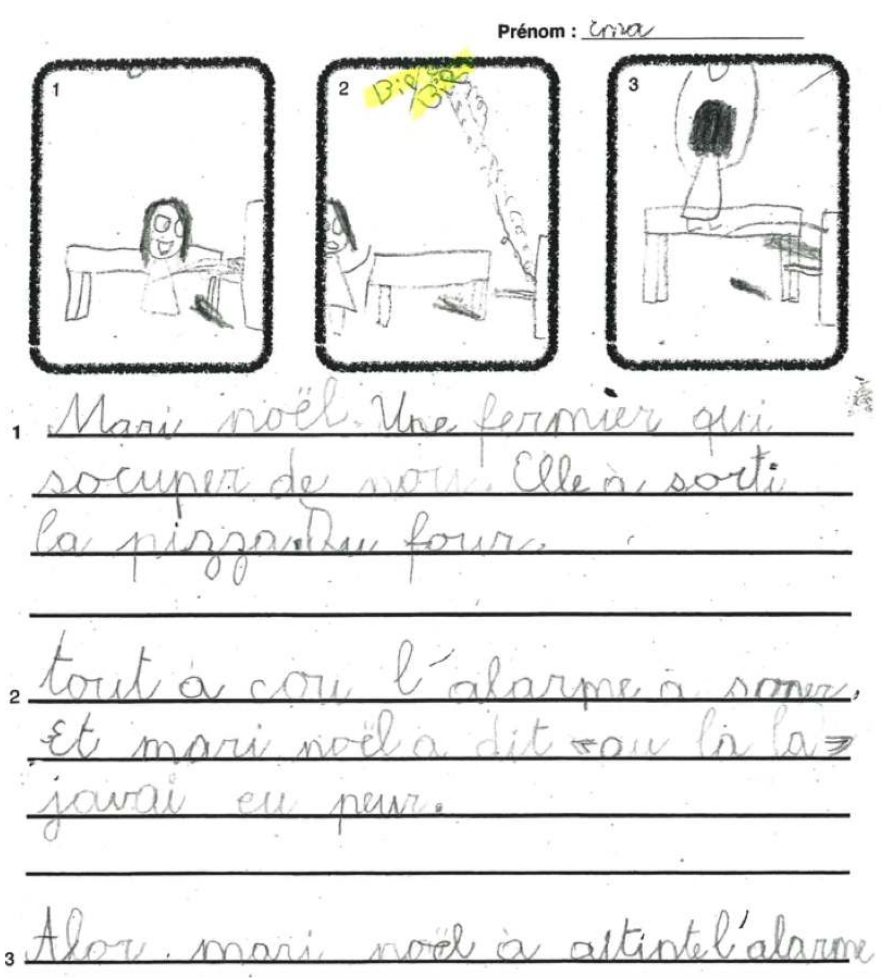

\section{RÉSUMÉS}

La didactique du français à l'École primaire romande est construite en référence aux genres, lesquels constituent l'arrière plan théorique du Plan d'Études Romand (PER, 2010). Selon cette posture épistémologique, le modèle didactique du genre, appuyé sur une description des principales caractéristiques d'un genre donné, occupe une place nodale dans le processus de transposition didactique. Cette modélisation s'effectue en référence au PER, dans lequel il est recommandé d'aborder tous les regroupements de genre dès le premier cycle $(1 \mathrm{H}-4 \mathrm{H})$ tandis qu'au cycle 2 chacun d'eux fera l'objet d'un travail à deux reprises. Ceci signifie que chaque année il faut aborder au moins trois regroupements de genre pour se conformer à la conception spiralaire des apprentissages préconisée. Nous analysons trois productions écrites d'élèves de $4 \mathrm{H}$ ( $4^{\mathrm{e}}$ HarmoS, classe de fin du cycle 1 romand/CE1, fin du cycle 2 en France) et leurs productions des années antérieures $(2 \mathrm{H}$ et $3 \mathrm{H})$ concernant ce même genre. Quelles traces d'un déjà là dans les choix linguistiques, discursifs et stylistiques des élèves peut-on repérer? Les élèves se distancient-ils ou non d'un schéma prototypique «simplification de la réalité réduite à des descripteurs » et font-ils preuve de capacité créatrice, mentionnée également dans le PER ?

In romand Switzerland, french didactic for Primary school is built in reference to "genres", which constitute the theoretical background of the curriculum "Plan d'Études Romand" (PER, 2010). The didactic model of "genre" takes a nodal place in the process of didactic transposition. The PER recommend to teach all groups of "genre" from $1 \mathrm{H}$ to $4 \mathrm{H}$ (cycle 1 ). In cycle 2 , it is necessary to teach every year at least three "groups of genre" to conform to the spiral learning 
prescribed. We analyze three pupils' writing (4 HarmoS, CE1 in France) and their previous writing $(2 \mathrm{H}$ and $3 \mathrm{H})$. What substratum in the linguistic, discursive and stylistic pupils' choices? Can they stand back from a prototypical process "simplification of the reality reduced to descriptors" and do they have creative capacity?

INDEX

Mots-clés : genre, modèle didactique, suivi longitudinal

Keywords : genre, didactic model, longitudinal follow-up

\section{AUTEURS}

VÉRONIQUE BOURHIS

Université de Cergy Pontoise, EMA, F-95000, France

\section{DANIÈLE FROSSARD}

Haute École Pédagogique, $\mathrm{CH}-1014$, Suisse

\section{MARTINE PANCHOUT}

Haute École Pédagogique, CH-1014, Suisse 